\title{
Predictors of atrial fibrillation in hypertrophic cardiomyopathy
}

\author{
Oliver P Guttmann ${ }^{1}$, Menelaos Pavlou ${ }^{2}$, Constantinos O’Mahony ${ }^{1}$, Lorenzo Monserrat ${ }^{3}$, Aristides \\ Anastasakis $^{4}$, Claudio Rapezzi ${ }^{5}$, Elena Biagini ${ }^{5}$, Juan Ramon Gimeno ${ }^{6}$, Giuseppe Limongelli ${ }^{7}$, Pablo \\ Garcia-Pavia $^{8}$, William J. McKenna ${ }^{1}$, Rumana Z. Omar ${ }^{2,9}$ and Perry M. Elliott ${ }^{1 *}$, for the Hypertrophic \\ Cardiomyopathy Outcomes Investigators
}

${ }^{1}$ University College London Institute for Cardiovascular Science and Barts Heart Centre, St. Bartholomew's Hospital, W Smithfield, London EC1A 7BE, United Kingdom

${ }^{2}$ Department of Statistical Science, University College London, Gower St, London WC1E 6BT, UK

${ }^{3}$ Cardiology Department and Research Unit, A Coruña University Hospital, Galician Health Service, Spain;

${ }^{4}$ Unit of Inherited Cardiovascular Diseases, 1st Department of Cardiology, University of Athens, 99 Michalakopoulou St, Athens 11527, Greece;

${ }^{5}$ Institute of Cardiology, Department of Specialised, Experimental and Diagnostic Medicine, University of Bologna, Via Massarenti 9, Bologna 40138, Italy;

${ }^{6}$ Cardiac Department, University Hospital Virgen Arrixaca, Murcia-Cartagena s/n. El Palmar, Murcia, 30120, Spain;

${ }^{7}$ Monaldi Hospital, Second University of Naples, Via Leonardo Bianchi 1, Naples 80131, Italy;

${ }^{8}$ Heart Failure and Inherited Cardiac Diseases Unit, Hospital Universitario Puerta del Hierro Majadahonda, Manuel de Falla, 1, 28222, Madrid, Spain; and

${ }^{9}$ Biostatistics Group, University College London Hospitals/University College London Clinical Research Centre, University College London, Gower St., London WC1E 6BT, UK;

Corresponding address:

Professor Perry M. Elliott, St. Bartholomew's Hospital, West Smithfield, London. EC1A7BE.

Email: perry.elliott@ucl.ac.uk; Tel: ++44 207377 7000; Fax: ++44 2073777000 


\begin{abstract}
Objectives: Atrial fibrillation (AF) is associated with increased morbidity and mortality in patients with hypertrophic cardiomyopathy (HCM). The primary aim of this study (HCM Risk-AF) was to determine predictors of $\mathrm{AF}$ in a large multicentre cohort of patients with HCM. Exploratory analyses were performed to investigate the association between AF and survival and the efficacy of antiarrhythmic therapy in maintaining sinus rhythm.
\end{abstract}

Methods: A retrospective, longitudinal cohort of patients recruited between 1986 and 2008 in seven centres, was used to develop multivariable Cox regression models fitted with preselected predictors. HCM was defined as unexplained hypertrophy (maximum left ventricular wall thickness $\geq 15 \mathrm{~mm}$ or in accordance with published criteria for the diagnosis of familial disease). Twenty-eight percent of patients $(n=1171)$ had coexistent hypertension. The primary endpoint was paroxysmal, permanent or persistent AF detected on ECG, Holter monitoring or implantable device interrogation.

Results: Of $4248 \mathrm{HCM}$ patients without pre-existing AF, 740 (17.4\%) reached the primary endpoint. Multivariable Cox regression revealed an association between AF and female sex, age, LA diameter, NYHA class, hypertension and vascular disease. The proportion of patients with cardiovascular death at 10 years was $4.9 \%$ in the sinus rhythm (SR) group and $10.9 \%$ in the AF group (difference in proportions $=5.9 \% ; 95 \% \mathrm{CI}=[4.1 \%-7.8 \%])$. The proportion of patients with non-cardiovascular death at 10 years was $3.2 \%$ in the SR group and $5.9 \%$ in the AF group (difference in proportions $=2.8 \%$; $95 \% \mathrm{CI}=[0.1 \%-4.2 \%])$. An intention to treat propensity score analysis demonstrated that $\beta$-blockers, calcium channel antagonists and disopyramide initially maintained sinus rhythm during follow up, and their protective effect diminished with time. Amiodarone therapy did not prevent AF during follow up.

Conclusions: This study shows that patients with HCM who are at risk of AF development can be identified using readily available clinical parameters. The development of AF is associated with a poor prognosis but there was no evidence that antiarrhythmic therapy prevents of AF in the long-term. 


\section{Keywords}

atrial fibrillation; hypertrophic cardiomyopathy 


\section{Key messages}

\section{What is already known about this subject?}

$\mathrm{AF}$ is a common complication of HCM and a number of studies have suggested that it is associated with adverse clinical outcomes and reduced survival.

\section{What does this study add?}

In this study, $17.4 \%$ of patients developed AF during the follow up period. Multivariable Cox regression revealed an association between the occurrence of new onset $\mathrm{AF}$ and the following: female sex, age, LA diameter, NYHA class, hypertension and vascular disease. Patients with AF had a more than twofold increased risk of cardiovascular death at 10 years. An intention to treat analysis utilising propensity score suggested that $\beta$-blockers, calcium channel antagonists and disopyramide initially maintained sinus rhythm, but their protective effect diminished with time. Amiodarone therapy did not prevent AF during follow up.

\section{How might this impact on clinical practice?}

This study confirms the importance of atrial fibrillation in the natural history of HCM. Patients with risk factors for AF should undergo frequent ambulatory ECG monitoring to detect atrial arrhythmia and could be considered for early prophylactic anticoagulation. Common cardiovascular risk factors such as vascular disease and hypertension should be given equal priority to other disease-specific complications. 


\section{Introduction}

Hypertrophic cardiomyopathy (HCM) is a myocardial disorder characterised by left ventricular hypertrophy not explained solely by abnormal loading conditions ${ }^{1}$. Atrial fibrillation (AF) is a common complication of the disease and a number of studies have suggested that it is associated with adverse clinical outcomes and reduced survival ${ }^{2-5}$. In order to prevent AF and its sequelae, it is necessary to develop methods for stratifying patients into sub-groups that will derive most benefit from targeted therapeutic strategies. In a recent multicentre study (HCM Risk-CVA) we demonstrated that patients at high risk of thromboembolic stroke can be identified using a small number of simple clinical parameters including a history of $\mathrm{AF}^{6}$. The primary aim of this study was to determine predictors of new onset $\mathrm{AF}$ in the same cohort. Additional exploratory analyses were performed to investigate the effect of AF on mortality and the efficacy of antiarrhythmic therapy in the prevention of AF.

\section{Methods}

\section{Study design and overview}

This study uses data from a retrospective, multicentre longitudinal cohort (HCM Risk-CVA) ${ }^{6}$.

The study conforms to the principles of the Helsinki declaration. The sponsors of this study did not have a role in study design, data collection, analysis, and interpretation. O.G., M.P., R.O., and P.E. had access to all data and had final responsibility to submit the article. The authors from each participating centre guarantee the integrity of data from their institution. All investigators have agreed to the article as written.

\section{Study population and participating centres}

The study cohort has been described in detail in a previous manuscript on thromboembolism in HCM (HCM Risk-CVA) ${ }^{6}$. Subgroups of patients from this cohort have been reported in other recently published studies (supplementary table 1). . The study cohort consisted of all consecutively evaluated patients with HCM, followed at seven participating European centres: (i) The Heart Hospital, 
University College London, London, UK, (ii) A Coruña University Hospital, A Coruña, Spain, (iii) Unit of Inherited Cardiovascular diseases, 1st Department of Cardiology, University of Athens, Greece, (iv) Institute of Cardiology, University of Bologna, Italy, (v) University Hospital Virgen de la Arrixaca, Murcia, Spain, (vi) Monaldi Hospital, Second University of Naples, Italy and (vii) Hospital Universitario Puerta del Hierro, Madrid, Spain.

Only adult patients ( $\geq 16$ years of age) were studied. HCM was defined as a maximum left ventricular wall thickness $\geq 15 \mathrm{~mm}$ unexplained solely by loading conditions ${ }^{7}$ or in accordance with published criteria for the diagnosis of disease in relatives of patients with unequivocal disease ${ }^{8}$. Patients with known inherited metabolic diseases or syndromic causes of HCM were excluded from the study. For the study of AF predictors, patients with AF at first evaluation were excluded from the analysis.

\section{Ethics approval}

Ethics approval and written informed consent was obtained for each participating centre. Patients at A Coruña University Hospital (Spain), 1st Department of Cardiology, University of Athens (Greece), University Hospital Virgen de la Arrixaca (Spain), and Monaldi Hospital (Italy) provided written informed consent. The data collection at The Heart Hospital Hospital (UK), Universitario Puerta de Hierro (Spain) and at the Institute of Cardiology at the University of Bologna (Italy) have been approved by the appropriate local ethics committee.

\section{Patient assessment and data collection}

All patients underwent pedigree analysis, clinical assessment, physical examination, resting and ambulatory electrocardiography and transthoracic echocardiography as previously described ${ }^{6}$. Each participating centre collected data independently. Patients were reviewed every 6-12 months or earlier if there was a change in symptoms. 


\section{Clinical outcomes}

The primary outcome was paroxysmal, permanent or persistent AF detected on ECG, Holter monitoring or device interrogation (table 1). The secondary outcome was all-cause and cardiovascular mortality (defined as sudden cardiac death, heart failure related death, stroke related death and other cardiac death). Sudden cardiac death was defined as witnessed sudden death with or without documented ventricular fibrillation or death within 1 hour of new symptoms or nocturnal deaths with no antecedent history of worsening symptoms. ${ }^{9}$ Heart failure related death was defined as death preceded by signs and symptoms of heart failure or cardiogenic shock. Stroke related death was defined as any death preceded by stroke. Pulmonary embolism and myocardial infarction were included in other cardiovascular death. All other deaths were considered as non-cardiovascular. ${ }^{10}$ 
Table 1: Definition of pre-specified predictor variables assessed at baseline evaluation

\begin{tabular}{|c|c|c|}
\hline $\begin{array}{l}\text { Predictor } \\
\text { variable }\end{array}$ & Definition & Coding \\
\hline Sex & Male or female ${ }^{11}$ & $\begin{array}{l}\text { Binary, } \\
\text { male }=1 / \text { female }= \\
0\end{array}$ \\
\hline Age & Age at first evaluation in participating centres ${ }^{12}$ & $\begin{array}{l}\text { Continuous, } \\
\text { years }\end{array}$ \\
\hline AF & $\begin{array}{l}\text { Physician reported detection of paroxysmal, permanent of persistent } \\
\text { AF on ECG, Holter monitoring or device interrogation }{ }^{12 ; 13}\end{array}$ & $\begin{array}{l}\text { Binary, } \\
\text { yes }=1 / \text { no }=0\end{array}$ \\
\hline NYHA & NYHA class at first evaluation ${ }^{12}$ & $\begin{array}{l}\text { Categorical, 1,2 } \\
\text { and 3-4 }\end{array}$ \\
\hline LA & $\begin{array}{l}\text { Anterior-posterior left atrial diameter determined by } 2 \mathrm{D} \\
\text { echocardiography in the parasternal long axis plane at time of first } \\
\text { evaluation }{ }^{11}\end{array}$ & Continuous, $\mathrm{mm}$ \\
\hline MWT & $\begin{array}{l}\text { The greatest LV wall thickness measured at the level of the mitral } \\
\text { valve, papillary muscles, and apex in the parasternal short-axis plane } \\
\text { using 2-D echocardiography at time of evaluation }{ }^{9}\end{array}$ & Continuous, $\mathrm{mm}$ \\
\hline FS & $\begin{array}{l}\text { LV end-diastolic dimension-LV end-systolic dimension)/ LV end- } \\
\text { diastolic dimension measured by M-Mode or } 2 \mathrm{D} \text { echocardiography at } \\
\text { time of evaluation }{ }^{11}\end{array}$ & Continuous, \% \\
\hline $\begin{array}{l}\text { LVOT } \\
\max \end{array}$ & $\begin{array}{l}\text { The maximum LV outflow gradient determined at rest and with } \\
\text { Valsalva provocation (irrespective of concurrent medical treatment) } \\
\text { using pulsed and continuous wave Doppler from the apical three- and } \\
\text { five-chamber views. Peak outflow tract gradients were determined } \\
\text { using the modified Bernoulli equation: Gradient }=4 \mathrm{~V}^{2} \text {, where } \mathrm{V} \text { is the } \\
\text { peak aortic outflow velocity }{ }^{14}\end{array}$ & $\begin{array}{l}\text { Continuous, } \\
\mathrm{mmHg}\end{array}$ \\
\hline $\begin{array}{l}\text { Hyperten } \\
\text { sion }\end{array}$ & $\begin{array}{l}\text { Diagnosis of hypertension prior to first evaluation, as per } \mathrm{CHA}_{2} \mathrm{DS}_{2-} \\
\text { VASc score }{ }^{13}\end{array}$ & $\begin{array}{l}\text { Binary, } \\
\text { yes }=1 / \text { no }=0\end{array}$ \\
\hline Diabetes & $\begin{array}{l}\text { Diagnosis of diabetes prior to first evaluation, as per } \mathrm{CHA}_{2} \mathrm{DS}_{2}-\mathrm{VASc} \\
\text { score }{ }^{13}\end{array}$ & $\begin{array}{l}\text { Binary, } \\
\text { yes }=1 / \text { no }=0\end{array}$ \\
\hline $\begin{array}{l}\text { Vascular } \\
\text { disease }\end{array}$ & $\begin{array}{l}\text { Myocardial infarction, complex aortic plaque and peripheral arterial } \\
\text { disease, as per } \mathrm{CHA}_{2} \mathrm{DS}_{2} \text {-VASc score }{ }^{13}\end{array}$ & $\begin{array}{l}\text { Binary, } \\
\text { yes }=1 / \text { no }=0\end{array}$ \\
\hline
\end{tabular}

AF: atrial fibrillation, NYHA: New York Heart Association Functional classification, LA: Left atrial size, MWT: Maximal wall thickness, FS: Fractional shortening, LVOT max: maximum LV outflow gradient

\section{Selection of predictors and coding}

Candidate predictor variables were selected following a review of the literature completed in September $2012{ }^{15}$. Clinical parameters were used as pre-specified predictor variables only when associated with AF in at least one published study. All parameters used in the final model were uniformly defined in all participating centres. In addition to these variables, predictors known to be 
associated with $\mathrm{AF}$ in the general population such as hypertension, diabetes mellitus and vascular disease were considered (table 1). All predictors were determined at baseline evaluation.

\section{Sample size}

A minimum of $10 \mathrm{AF}$ endpoints are required per coefficient estimated by the model to ensure that the regression coefficients are estimated with adequate precision. The $740 \mathrm{AF}$ endpoints observed in this cohort over a 10 year follow-up period allow the estimation of all 11 candidate regression coefficients (as shown in table 1) in a multivariable regression model and also adjust for possible centre effects.

\section{General statistical methods}

STATA (version 12) and R (version 3.0) were used for the statistical analyses. For descriptive results, variables are expressed as mean \pm standard deviation $(\mathrm{SD})$, median and interquartile range (IQR) or counts and percentages as appropriate. The follow-up time for each patient was calculated from the date of their first evaluation at participating centres to the date of the relevant endpoint or to the date of their most recent evaluation. The annual event rate was calculated by dividing the number of patients reaching the endpoint by the total follow-up period for that endpoint. The Kaplan-Meier method was used to estimate the cumulative probability for the occurrence of an outcome.

\section{Missing data}

The characteristics of patients with missing information were compared to those of patients with complete information to investigate bias due to missing data. Logistic regression was used to identify the predictors of missingness. Data were assumed to be missing at random and values for the missing predictors were imputed using multiple imputation techniques based on chained equations ${ }^{16}$. The multiple imputation model included all predictors of missingness, the outcome, all pre-specified predictors of the risk model, and the estimate of the cumulative hazard function ${ }^{17}$. Rubin's rules were 
used to combine the estimates from 30 imputed data sets ${ }^{18}$. We also carried out a complete case analysis, excluding patients with missing observations in any of the candidate predictor variables.

\section{Survival analysis}

Univariable and multivariable Cox regression models were fitted and tested for non-linearity of continuous predictors by inclusion of quadratic terms. The final fully adjusted Cox regression model for $\mathrm{AF}$ was developed using backwards elimination and a $15 \%$ significance level was used to select the final predictors ${ }^{19}$. The proportional hazards assumption was investigated using Schoenfeld residuals ${ }^{20}$. A sensitivity analysis for centre effects was performed by including centre in the model. As part of the sensitivity analysis a complete case analysis of patients with no missing data.

Cardiovascular and non-cardiovascular mortality was compared in patients with AF and SR at any time during the follow up period of ten years. Patients with AF at first evaluation were included for this analysis.

A propensity score analysis was used to investigate clinical outcomes in patients receiving $\beta$ adrenoreceptor blockers, non-dihydropyridine calcium channel antagonists, disopyramide and amiodarone. If a patient received treatment at any time prior to the event it was assumed that they continued on this medication until the endpoint of the study was reached or the end of follow up. The effect on development of $\mathrm{AF}$ was investigated. A sub-analysis was performed to assess the relationship between risk of AF and LA size. 


\section{Results}

The initial study cohort comprised 5104 patients. Of these, 197 were only seen once at baseline evaluation and so were excluded from all subsequent analyses. The remaining cohort of 4907 patients was used to model the relation between AF and survival; 659 patients had AF prior to first evaluation and were excluded from the analysis of new AF events (table 2). Missing data per variable are presented in supplementary table 2. Clinical characteristics of the patients with AF at baseline evaluation, who were excluded, are presented in supplementary table 3.

Table 2: Clinical characteristics of whole cohort and in patients with and without AF

\begin{tabular}{ccccccc}
\hline & whole cohort & \multicolumn{3}{c}{ SR } & AF \\
\hline variable & mean/n & SD/\% & mean/n & SD/\% & mean/n & SD/\% \\
Age & 47.73 & 16.3 & 46.73 & 16.52 & 52.48 & 14.34 \\
LA $(\mathrm{mm})$ & 43.1 & 7.2 & 42.12 & 6.8 & 47.69 & 7.26 \\
MWT $(\mathrm{mm})$ & 19.47 & 5.23 & 19.33 & 5.27 & 20.14 & 5.02 \\
FS \% & 0.41 & 0.09 & 0.41 & 0.09 & 0.41 & 0.1 \\
LVOT max & 32.67 & 41.49 & 31.11 & 40.4 & 39.75 & 45.43 \\
Female & 1504 & 0.35 & 1214 & 0.35 & 290 & 0.39 \\
NYHA II & 1365 & 0.34 & 1057 & 0.31 & 308 & 0.43 \\
NYHA III,IV & 377 & 0.09 & 269 & 0.08 & 108 & 0.15 \\
Hypertension & 1171 & 0.28 & 914 & 0.27 & 257 & 0.36 \\
Diabetes & 225 & 0.06 & 168 & 0.06 & 57 & 0.09 \\
Vascular & 66 & 0.02 & 41 & 0.02 & 25 & 0.04 \\
disease & & & & & &
\end{tabular}

SD: Standard deviation, SR: sinus rhythm, n: Number, LA: Left atrial size, MWT: Maximal wall thickness, FS: Fractional shortening, LVOT max: maximum LV outflow gradient, AF: atrial fibrillation, NYHA: New York Heart Association Functional classification, FH SCD: Family history of sudden cardiac death

\section{Predictors of AF}

AF events during follow-up

During a follow up period of 22,743 patient years (median 5.4 years), 740/4248 (17.4\%) patients who were in sinus rhythm at baseline reached the primary endpoint within 10 years from first evaluation $(\mathrm{n}=57.8 \%$ paroxysmal $\mathrm{AF}$ and $\mathrm{n}=42.2 \%$ permanent/persistent $\mathrm{AF}$ ). The 5 and 10 year cumulative incidence rates of $\mathrm{AF}$ were $16.0 \%$ (95\% CI $14.6 \%-17.5 \%$ ) and $33.3 \%$ (95\% CI $30.7 \%-36.1 \%$ ), 
respectively. The clinical characteristics of patients with and without AF during the follow up period are shown in table 2 .

\section{Univariable and multivariable analyses for primary endpoint}

The models for predicting new onset AF were developed using the 740 events within the first 10 years of follow-up. Univariable analyses are shown in table 3. Maximal LV wall thickness and LA size had a nonlinear association with $\mathrm{AF}$ and so a quadratic term was included in the multivariable analysis.

Multivariable Cox regression showed an association between new onset $\mathrm{AF}$ and the following: female sex, age, LA diameter (and its quadratic term), NYHA class II, NYHA class III/IV, hypertension and vascular disease as shown in table 3 .

Sensitivity analyses

A model including centre was also fitted as part of a sensitivity analysis. There was a significant centre effect on AF; however there was no change in the estimates of the hazard ratios (supplementary table 4). Clinical characteristics according to centre are displayed in supplementary figure 5. As part of the sensitivity analysis a complete case analysis of patients with no missing data $(n=3831 ; 676$ events) was also performed. This analysis showed an association between new onset AF and the same variables except for vascular disease and hypertension. This could be due to smaller sample of patients included in the analysis. 
Table 3: Univariable and multivariable analysis for predictors of $\mathrm{AF}$ in $\mathrm{HCM}$

\begin{tabular}{cccc} 
Univariable analysis & & & \\
\hline Predictor & HR & Cl & $\mathbf{p}$ \\
Female & 1.27 & $1.10-1.47$ & $<0.001$ \\
Age (10y increment) & 1.35 & $1.29-1.41$ & $<0.001$ \\
NYHA II & 1.75 & $1.49-2.05$ & $<0.001$ \\
NYHA III,IV & 2.96 & $2.37-3.69$ & $<0.001$ \\
LA (5mm increment) & 1.6 & $1.53-1.68$ & $<0.001$ \\
MWT (mm) & 1.02 & $1.01-1.03$ & $<0.001$ \\
FS (\%) & 0.87 & $0.38-2.01$ & 0.75 \\
LVOT max (mmHg) & 1.01 & $1.00-1.01$ & $<0.001$ \\
LVEDD (mm) & 1.02 & $1.01-1.03$ & $<0.001$ \\
LVESD (mm) & 1.01 & $1.00-1.03$ & 0.02 \\
Hypertension & 1.72 & $1.48-2.01$ & $<0.001$ \\
Diabetes & 1.52 & $1.16-1.99$ & $<0.001$ \\
Vascular disease & 2.4 & $1.61-3.58$ & $<0.001$ \\
Analyses with non-linear & & & \\
terms & & & \\
MWT (mm) & 1.21 & $1.11-1.31$ & $<0.001$ \\
MWT2 & 1 & $0.99-1.00$ & $<0.001$ \\
LA5 & 3.11 & $1.98-4.87$ & $<0.001$ \\
LA5 & 0.97 & $0.94-0.99$ & $<0.001$ \\
& & &
\end{tabular}

\section{Multivariable analysis}

$\begin{array}{cccc}\text { Predictor } & \text { HR } & \mathbf{9 5 \%} \mathbf{C l} & \mathbf{P} \\ \text { Female } & 1.31 & 1.12-1.53 & 0.001 \\ \text { Age (in 10y increments) } & 1.21 & 1.15-1.28 & <0.001 \\ \text { NYHA II } & 1.29 & 1.10-1.51 & <0.001 \\ \text { NYHA III,IV } & 1.63 & 1.30-2.04 & <0.001 \\ \text { LA (in 5 mm increments) } & 2.51 & 1.60-3.94 & <0.001 \\ \text { LA (in 5mm increments) } & 0.98 & 0.98-1.35 & 0.06 \\ \text { Hypertension } & 1.15 & 0.90-1.96 & 0.1 \\ \text { Vascular disease } & 1.33 & 0.90-1.96 & 0.15\end{array}$

Age10: Hazard ratio for 10 year increments, NYHA: New York Heart Association Functional classification, LA5: Hazard ratio for left atrial size for 5mm increments, MWT: Maximal wall thickness, FS: Fractional shortening, LVOT max: maximum LV outflow gradient, LVEDD: Left ventricular end-diastolic dimension, LVESD: left ventricular end-systolic dimension, HR: Hazard ratio, p: p-value, $\mathrm{Cl}$ : $95 \%$ confidence interval, $\mathrm{MWT}$ and $\mathrm{MWT}^{2}$ in the last two rows of the table adjust for MWT and its square term and similarly for LA5. 


\section{Relation of LA size to AF}

Using the multivariable Cox regression model (table 3) we calculated the risk of AF for a range of values for left atrium size (the values of the other predictors in the model were fixed at their mean value). The predicted risks of $\mathrm{AF}$ were then plotted against the values for left atrium size (figure 1). There was a linear relationship between LA size and AF risk above a diameter of $40 \mathrm{~mm}$.

\section{Mortality in patients with AF and SR}

Patients with unknown date $(n=47)$ or cause of death $(n=25)$ were excluded from this analysis. The proportion of patients with cardiovascular death was $8.4 \%$ over the whole follow up period, $95 \% \mathrm{CI}=$ [7.6\%-9.2\%] (405 out of 4835 patients). The proportion over a 10 year follow up was $6.7 \%, 95 \% \mathrm{CI}=$ [5.9\%-7.3\%] (317 out of 4835 patients). The proportion of patients with non-cardiovascular death was $4.9 \%$ over the whole follow up period, $95 \% \mathrm{CI}=[4.2 \%-5.4 \%]$ (234 out of 4835 patients). The proportion over a 10 year follow up was $3.91 \%, 95 \% \mathrm{CI}=[3.4 \%-4.5 \%]$ (189 out of 4835 patients).

Patients in AF at any time were more likely to experience cardiovascular (10.9\% in the AF group versus $4.9 \%$ in the SR group (difference in proportions $=5.9 \% ; 95 \% \mathrm{CI}=[4.1 \%-7.8 \%]$ ) and noncardiovascular death $(5.9 \%$ in the AF group versus $3.2 \%$ in the SR group (difference in proportions= $2.8 \% ; 95 \% \mathrm{CI}=[0.1 \%-4.2 \%])$ than patients in SR over the 10 year follow up. There was an increased mortality in patients with permanent and persistent AF compared with paroxysmal AF (difference in proportions $=8.1 \% ; 95 \% \mathrm{CI}=[4.5 \%-11.7 \%])$

\section{Effect of antiarrhythmic drugs on clinical outcomes}

For each therapy, a Cox regression model for the time to AF adjusting for therapy and the propensity score was considered ${ }^{21}$. For disopyramide, beta-blocker and calcium channel antagonists the proportional hazards assumption was not satisfied, meaning that the effect of treatment on AF varies with time. To account for this effect we analysed the effect of therapy over time. Plots for the Schoenfeld residuals ${ }^{20}$ revealed a decreasing effect of therapy with time (for all three therapies). According to Schoenfeld plots a decreasing linear trend of the effect of therapy with time appeared 
plausible for disopyramide and beta-blockers. A linear trend could not be assumed for calcium channel antagonists and hence a log-transformation for time was used.

Disopyramide, beta-blocker, calcium-channel antagonists initially had a protective effect $(H R<1)$ but this effect seems to be attenuated with time (figures 2). This could be explained by disease progression. A further reason could be the fact that the propensity score accounts for the probability of patients receiving therapy given their characteristics at baseline, but does not account for the probability of receiving therapy for patients whose characteristics changed during follow up and the patients received therapy or switched from one therapy to another after baseline.

The effect of amiodarone was not statistically significant $(\mathrm{HR}=1.12 ; 95 \% \mathrm{CI}=[0.89-1.36])$ and did not appear to vary over time. In light of these findings, we examined the number of patients on amiodarone in the absence of a diagnosis of $\mathrm{AF}$ at first evaluation. Out of 311 patients receiving amiodarone at first evaluation only 23 had a prior diagnosis of AF.

\section{Discussion}

This study confirms the high prevalence of AF in HCM and reveals an association between AF and atrial size, NYHA class and age $3 ; 4 ; 15$. For the first time in a HCM population, we demonstrate an association between AF and several other patient characteristics including sex, hypertension and vascular disease.

Prevalence, Incidence of $A F$ and mortality

The prevalence and incidence of AF and mortality are in line with previous cohort studies in patients with $\mathrm{HCM}^{4 ; 5 ; 22 ; 23}$. Evidence for the detrimental effect of $\mathrm{AF}$ on survival is limited in $\mathrm{HCM}{ }^{4 ; 15}$ but in this study we show a significant association with cardiovascular mortality reflecting a higher proportion of heart failure and stroke related death in the AF group. It is possible that, in patients who die of heart failure, the onset of AF accelerates disease progression. Alternatively, AF may simply be a marker that identifies patients with advanced disease who are already on an accelerated trajectory to ventricular failure. The trend towards increased non cardiovascular mortality in the AF group is likely to represent a complex interplay between some miscoding of deaths and the adverse consequences of cardiac disease and therapy on general health. 


\section{Effect of antiarrhythmic therapy on AF development}

There are no prospective randomised trials of medical therapy for AF in HCM and only a very small number of observational studies assessing the effect of antiarrhythmic therapy on the maintenance of sinus rhythm ${ }^{24 ; 25}$. In this study a propensity score analysis demonstrated that $\beta$-blockers, calcium channel antagonists and disopyramide at baseline initially prevented AF for the first two to three years of follow up, but their protective effect then diminished. The retrospective nature of the analysis and the intention to treat analysis means that these findings should be interpreted cautiously, but it is likely that this loss of a therapeutic effect reflects the inexorable progression of disease. Contrary to expectations, amiodarone therapy did not have a protective effect on the development of AF. However, a large proportion of patients on amiodarone did not have a history of AF at first evaluation and we speculate that the apparently neutral effect of the drug might be explained by its historical use to prevent sudden cardiac death in patients with non-sustained ventricular tachycardia (NSVT) as this group of patients may have more myocardial fibrosis and advanced disease and thus be less likely to respond ${ }^{26 ; 27}$.

\section{Clinical implications}

This study confirms the importance of atrial fibrillation in the natural history of $\mathrm{HCM}{ }^{1}$. The findings in this and our previous study on stroke in $\mathrm{HCM}^{6}$ suggest that patients with risk factors for AF should undergo frequent ambulatory ECG monitoring to detect atrial arrhythmia and could be considered for early prophylactic anticoagulation. The identification of associations with common cardiovascular risk factors such as vascular disease and hypertension is not by itself that surprising, but shows that management of these risk factors should be given equal priority to other disease-specific complications. In spite of its limitations, the propensity score analysis for antiarrhythmic therapy suggests that rhythm control strategies for patients with AF and HCM are suboptimal and require revaluation in prospective randomised trials. 


\section{Limitations}

The predictors identified in this study may not apply to patients with different characteristics. In particular, the study excludes paediatric patients (less than 16) and patients with metabolic or syndromic disorders in whom other disease characteristics may influence the risk of AF development.

Changes in imaging technology, rhythm monitoring and device therapy may be relevant over the long follow up period.

The significant centre effect shown in a sensitivity analysis can be explained by the difference in some of the clinical characteristics of the participating cohorts including age, follow-up time and prevalence of atrial fibrillation during the follow up period. Further work is necessary to understand this, but it might reflect systematic differences in referral patterns.

\section{Conclusions}

Readily available clinical parameters can be used to identify patients at high risk of AF who require more frequent monitoring and early anticoagulation. In the exploratory analyses, AF was associated with increased cardiovascular and non-cardiovascular mortality and there was no evidence for a sustained beneficial effect of antiarrhythmic therapy on the development of atrial arrhythmia. 


\section{Acknowledgements}

\section{Funding}

This study was funded by the British Heart Foundation and by the National Institute for Health Research University College London Hospitals Biomedical Research Centre. It was also partially supported by the Red Investigación Cardiovascular from the Instituto de Salud Carlos III, Spanish Ministry of Health [grants RD12/0042/0049, RD12/0042/0066, and RD12/0042/0069] and Project $\mathrm{N}^{\circ}$ PI11/02604, integrated in the National Plan for Scientific Research, Development and Technological Innovation 2008-2011 and funded by the ISCIII- General Subdirection of Assessment and Promotion of the Research - European Regional Development Fund (FEDER) "A way of making Europe”. OPG received research support from the British Heart Foundation (FS/12/86/29841) and the National Institute for Health Research University College London Hospitals Biomedical Research Centre.

\section{Authors' contributions}

OPG designed the study, collected and interpreted the data, carried out the descriptive statistical analysis, and wrote the article. P.M.E. designed the study, interpreted the data, and wrote the article. R.Z.O. was involved in study design, led the statistical aspects of the risk modelling and wrote the article. M.P. was involved in the study design and carried out the statistical analysis. C.O'M., L.M., A.A., E.B., J.R.G., G.L., P.G.P. and C.R. collected and interpreted the data and critically reviewed the manuscript. W.J.M. was involved in the drafting of the article and revising it critically for important intellectual content.

Antonis Pantazis, Maite Tome-Esteban, Shaughan Dickie, Xusto Fernandez, Angela Lopez, Vasiliki Vlagkouli, Antonio Romero, Andrea Buono and Maria Gallego-Delgado and Marta Cobo-Marcos were involved in data collection and interpretation.

The Corresponding Author has the right to grant on behalf of all authors and does grant on behalf of all authors, an exclusive licence (or non-exclusive for government employees) on a worldwide basis to the BMJ Publishing Group Ltd and its Licensees to permit this article (if accepted) to be published in HEART editions and any other BMJPGL products to exploit all subsidiary rights. 
Reference List

(1) Elliott PM, Anastasakis A, Borger MA et al. 2014 ESC Guidelines on diagnosis and management of hypertrophic cardiomyopathy: the Task Force for the Diagnosis and Management of Hypertrophic Cardiomyopathy of the European Society of Cardiology (ESC). Eur Heart J 2014;35:2733-2779.

(2) Cecchi F, Olivotto I, Betocchi S et al. The Italian Registry for hypertrophic cardiomyopathy: a nationwide survey. Am Heart J 2005;150:947-954.

(3) Maron BJ, Olivotto I, Bellone P et al. Clinical profile of stroke in 900 patients with hypertrophic cardiomyopathy. J Am Coll Cardiol 2002;39:301-307.

(4) Olivotto I, Cecchi F, Casey SA, Dolara A, Traverse JH, Maron BJ. Impact of Atrial Fibrillation on the Clinical Course of Hypertrophic Cardiomyopathy. Circulation 2001;104:2517-2524.

(5) Cecchi F, Olivotto I, Montereggi A, Santoro G, Dolara A, Maron BJ. Hypertrophic cardiomyopathy in Tuscany: clinical course and outcome in an unselected regional population. J Am Coll Cardiol 1995;26:1529-1536.

(6) Guttmann OP, Pavlou M, O'Mahony $C$ et al. Prediction of thrombo-embolic risk in patients with hypertrophic cardiomyopathy (HCM Risk-CVA). Eur J Heart Fail 2015;17:837-845.

(7) Elliott P, Andersson B, Arbustini E et al. Classification of the cardiomyopathies: a position statement from the european society of cardiology working group on myocardial and pericardial diseases. Eur Heart J 2008;29:270-276.

(8) McKenna WJ, Spirito P, Desnos M, Dubourg O, Komajda M. Experience from clinical genetics in hypertrophic cardiomyopathy: proposal for new diagnostic criteria in adult members of affected families. Heart 1997;77:130-132.

(9) Elliott PM, Poloniecki J, Dickie $S$ et al. Sudden death in hypertrophic cardiomyopathy: identification of high risk patients. J Am Coll Cardiol 2000;36:2212-2218.

(10) Elliott PM, Gimeno JR, Thaman R et al. Historical trends in reported survival rates in patients with hypertrophic cardiomyopathy. Heart 2006;92:785-791.

(11) Kubo $T$, Kitaoka $H$, Okawa $M$ et al. Clinical impact of atrial fibrillation in patients with hypertrophic cardiomyopathy. Results from Kochi RYOMA Study. Circ J 2009;73:1599-1605.

(12) Maron BJ, Olivotto I, Bellone P et al. Clinical profile of stroke in 900 patients with hypertrophic cardiomyopathy. J Am Coll Cardiol 2002;39:301-307.

(13) Camm AJ, Lip GY, De CR et al. 2012 focused update of the ESC Guidelines for the management of atrial fibrillation: an update of the 2010 ESC Guidelines for the management of atrial fibrillation--developed with the special contribution of the European Heart Rhythm Association. Europace 2012;14:1385-1413.

(14) Gimeno JR, Tome-Esteban M, Lofiego $C$ et al. Exercise-induced ventricular arrhythmias and risk of sudden cardiac death in patients with hypertrophic cardiomyopathy. Eur Heart J 2009;30:2599-2605. 
(15) Guttmann OP, Rahman MS, O'Mahony C, Anastasakis A, Elliott PM. Atrial fibrillation and thromboembolism in patients with hypertrophic cardiomyopathy: systematic review. Heart 2014;100:465-472.

(16) van BS, Boshuizen HC, Knook DL. Multiple imputation of missing blood pressure covariates in survival analysis. Stat Med 1999;18:681-694.

(17) White IR, Royston P, Wood AM. Multiple imputation using chained equations: Issues and guidance for practice. Stat Med 2011;30:377-399.

(18) Rubin D. Multiple Imputation for Nonresponse in Surveys. New York: John Wiley and Sons, 1987.

(19) Ambler G, Seaman S, Omar RZ. An evaluation of penalised survival methods for developing prognostic models with rare events. Stat Med 2012;31:1150-1161.

(20) Schoenfeld DAVI. Partial residuals for the proportional hazards regression model. Biometrika 1982;69:239-241.

(21) D'Agostino RB, Jr. Propensity scores in cardiovascular research. Circulation 2007;115:23402343.

(22) Siontis KC, Geske JB, Ong K, Nishimura RA, Ommen SR, Gersh BJ. Atrial fibrillation in hypertrophic cardiomyopathy: prevalence, clinical correlations, and mortality in a large highrisk population. J Am Heart Assoc 2014;3:e001002.

(23) Guttmann OP, Rahman MS, O'Mahony C, Anastasakis A, Elliott PM. Atrial fibrillation and thromboembolism in patients with hypertrophic cardiomyopathy: systematic review. Heart 2013.

(24) Cecchi F, Olivotto I, Montereggi A, Santoro G, Dolara A, Maron BJ. Hypertrophic cardiomyopathy in Tuscany: clinical course and outcome in an unselected regional population. J Am Coll Cardiol 1995;26:1529-1536.

(25) Tendera M, Wycisk A, Schneeweiss A, Polonski L, Wodniecki J. Effect of sotalol on arrhythmias and exercise tolerance in patients with hypertrophic cardiomyopathy. Cardiology 1993;82:335-342.

(26) Shiozaki AA, Senra T, Arteaga E et al. Myocardial fibrosis detected by cardiac CT predicts ventricular fibrillation/ventricular tachycardia events in patients with hypertrophic cardiomyopathy. J Cardiovasc Comput Tomogr 2013;7:173-181.

(27) O'Hanlon R, Grasso A, Roughton M et al. Prognostic significance of myocardial fibrosis in hypertrophic cardiomyopathy. J Am Coll Cardiol 2010;56:867-874. 


\section{Figure legends}

Figure 1: Relationship of LA size with risk of AF

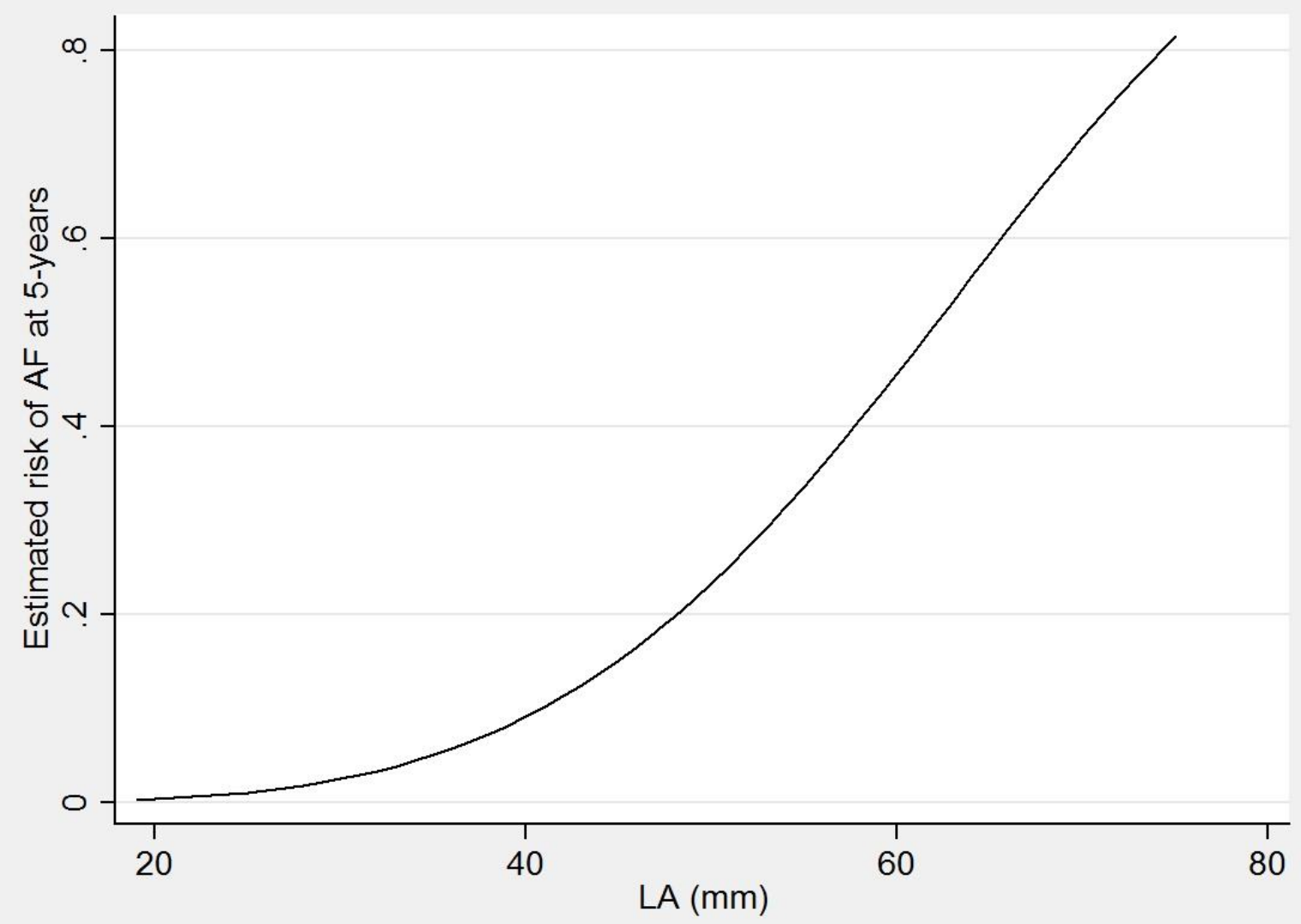


Figure 2: $\quad$ Propensity score analysis for development of AF over 5 year follow up period in treatment and non-treatment group (time-varying effect of $\beta$-blocker, Ca-channel antagonist, disopyramide)
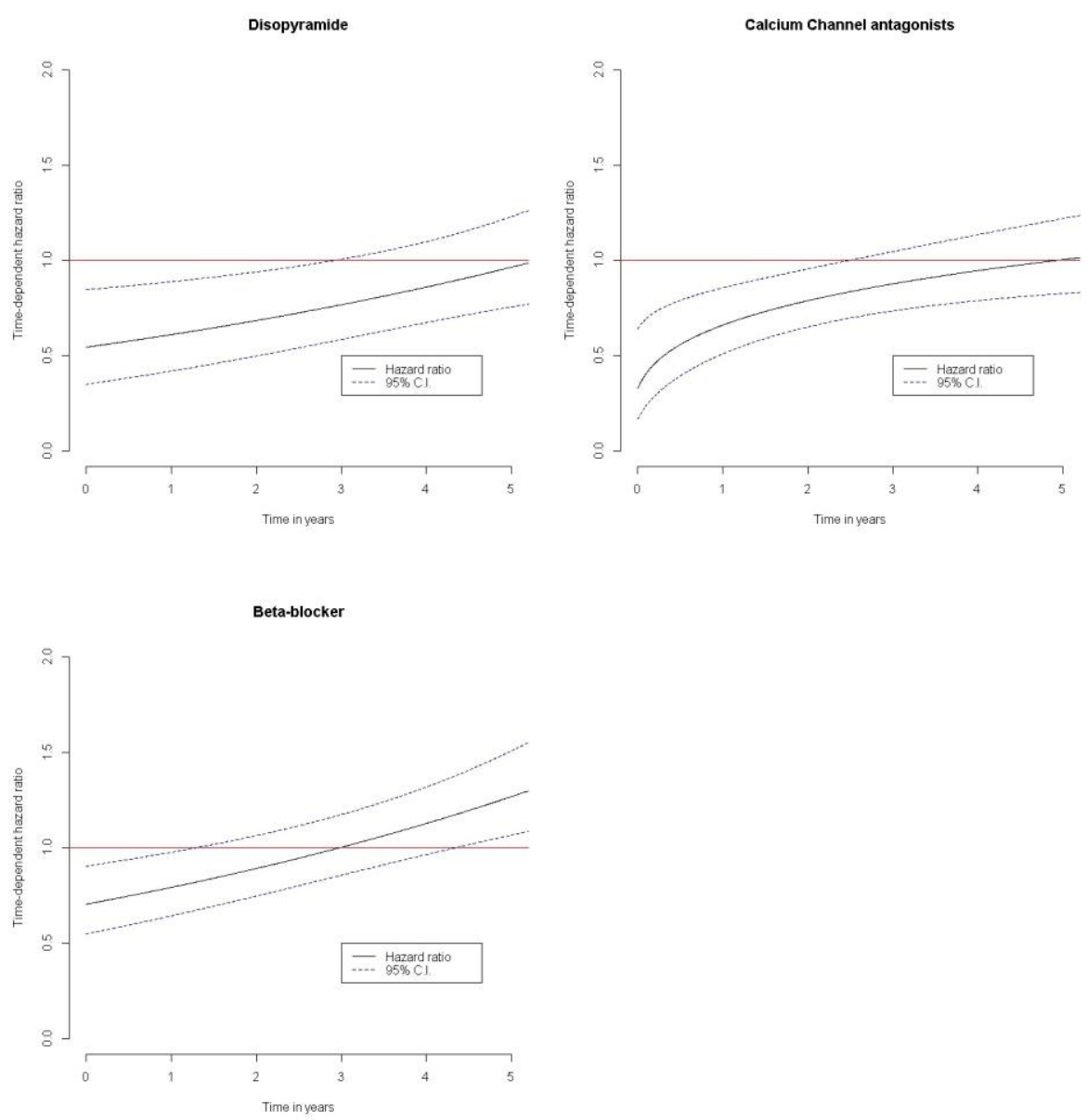